\title{
Endovascular therapy of arteriovenous malformation in a male patient with severe post-coital pelvic pain
}

\author{
Bartosz Zabicki ${ }^{1,2 A, B, C, D, E, F, G}$, Marte Johanne V. Holstad ${ }^{10, E, F}$, Nattakarn Limphaibool ${ }^{10, E, F}$, Robert Juszkat ${ }^{10, G}$ \\ 'Department of Diagnostic and Interventional Radiology, University Hospital No 1, Poznan University of Medical Sciences, Poland \\ ¿University Hospital Zielona Góra, Poland
}

\section{Abstract}

Purpose: Congenital pelvic arteriovenous malformations (AVMs) are high-flow vascular lesions consisting of abnormal shunts between arteries and veins within a nidus. The rare presentation and extensive network of vasculature contributes to the difficulty in effective treatment. Optimal therapeutic options are determined based on the clinical presentation, the location of the lesion, and possible complications.

Case report: A 24-year-old male patient with a history of recurrent pain following sexual intercourse presented with complaints of intense pelvic pain radiating to the perineal area. Computed tomography angiography (CTA) revealed a large venous aneurysm as an outflow vein of a right-sided pelvic AVM. Embolisation of the outflow veins was established along with direct percutaneous delivery of fibre coils and thrombin to the venous aneurysm of the AVM. With recurring symptoms and AVM recanalisation on angiography, another direct puncture and placement of pushable coils was made. Total AVM occlusion was achieved with no recanalisation on follow-up digital subtraction angiography (DSA), and the patient remained asymptomatic.

Conclusions: Endovascular embolisation of the nidus area may result in a complete occlusion of an AVM. Therefore, a thorough understanding of the vascular anatomy of the AVM is essential in choosing an effective embolisation strategy and to minimise the risk of possible complications.

Key words: endovascular procedure, embolisation, arteriovenous malformations, post-coital.

\section{Introduction}

Congenital arteriovenous malformations (AVMs) are high-flow vascular lesions consisting of abnormal shunts between arteries and veins within a nidus [1]. The pelvic location of this lesion is especially rare in males, usually not becoming symptomatic before early adulthood. Therapy centred around the obliteration of the AVM nidus can be difficult due to the complicated network of vessels. Endovascular therapy with embolisation, usually preferred over surgical treatment, still has a relatively high recurrence rate [2]. We report the stepwise treatment of a symptomatic congenital pelvic AVM presenting in a 24-year-old male utilising a combination of different endovascular techniques.

\section{Case report}

A 24-year-old male presented to the emergency department complaining of intense pelvic pain radiating to the perineal area, occurring as a result of sexual intercourse and ejaculation. At presentation, the patient rated the pain as 9 out of 10 points on the Visual Analogue Scale (VAS), refractory to nonsteroidal anti-inflammatory drugs (NSAIDs).

Correspondence address:

Dr. Bartosz Zabicki, Department of Diagnostic and Interventional Radiology, University Hospital No. 1, 1/2 Długa St., 61- 848 Poznań, Poland,

e-mail: bzabickigmail.com

Authors' contribution:

A Study design - B Data collection · C Statistical analysis · D Data interpretation - E Manuscript preparation · F Literature search · G Funds collection 


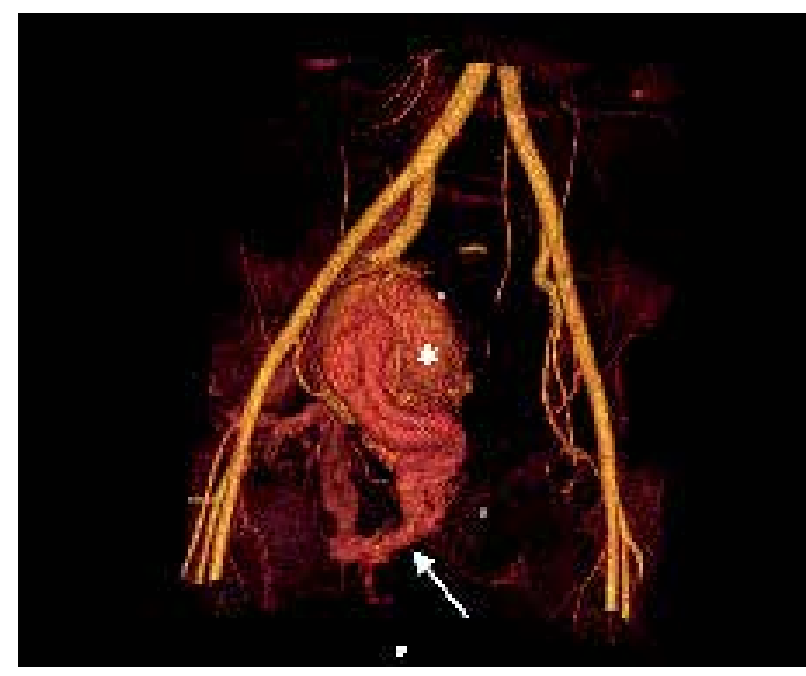

Figure 1. Computed tomography volume rendering reconstruction. Pelvic right-sided arteriovenous malformation. Multiple branches of the right internal iliac artery entangling and feeding the nidus located in the wall of an outflow venous aneurysm (asterisk). Multiple further outflow veins to the iliac venous system (arrow)

An abdominal ultrasound (US) was performed, which showed an aneurysm in the right pelvic area. Subsequent computed tomography angiography (CTA) confirmed the presence of a large venous aneurysm (approx. $10 \mathrm{~cm}$ in diameter), which transpired to be an outflow vessel of a predominantly right-sided pelvic AVM, supplied by multiple visceral branches of the right internal iliac artery (IIA) (Figure 1). The patient was transferred to the University Hospital and referred to emergent digital subtraction angiography (DSA). Access was acquired via the left femoral artery, and selective DSA of the right IIA depicted multiple vessels supplying the AVM (Figure 2). Selected feeding branches of the right internal iliac artery were catheterised with a Rebar 18 microcatheter (Medtronic) and embolised

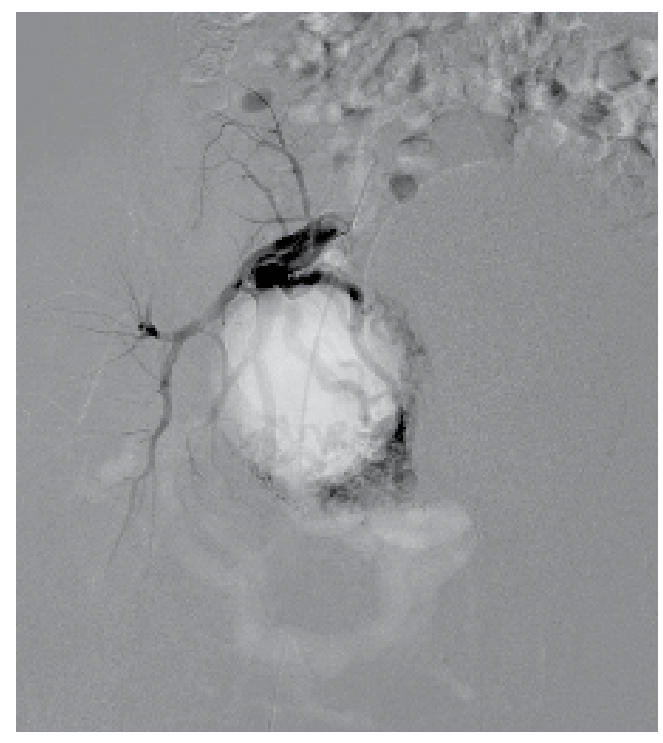

Figure 2. Selective digital subtraction angiography of the right internal iliac artery. Multiple arterial branches (in dark) supplying an aneurysmal outflow vein (in white)

using Squid-18 Peri embolic agent (EVOH in DMSO) (Balt/Emboflu). Minor symptomatic relief was achieved, but the AVM remained patent.

Five months later, the patient was readmitted with recurrence of pain, rated at 10 on the VAS. Angioarchitecture was re-evaluated and classified as IIIb based on the Yakes classification. Both arterial and venous accesses were performed. Due to a complicated venous tract, the venous aneurysm was achievable only with a microcatheter. Thus, only outflow veins were embolised with detachable coils (Concerto, Medtronic). US guidance was subsequently utilised to directly puncture and fill the venous aneurysm with pushable fibre coils (Nester, MRey coils, Cook Medical) (Figure 3A). Additionally, two ampoules
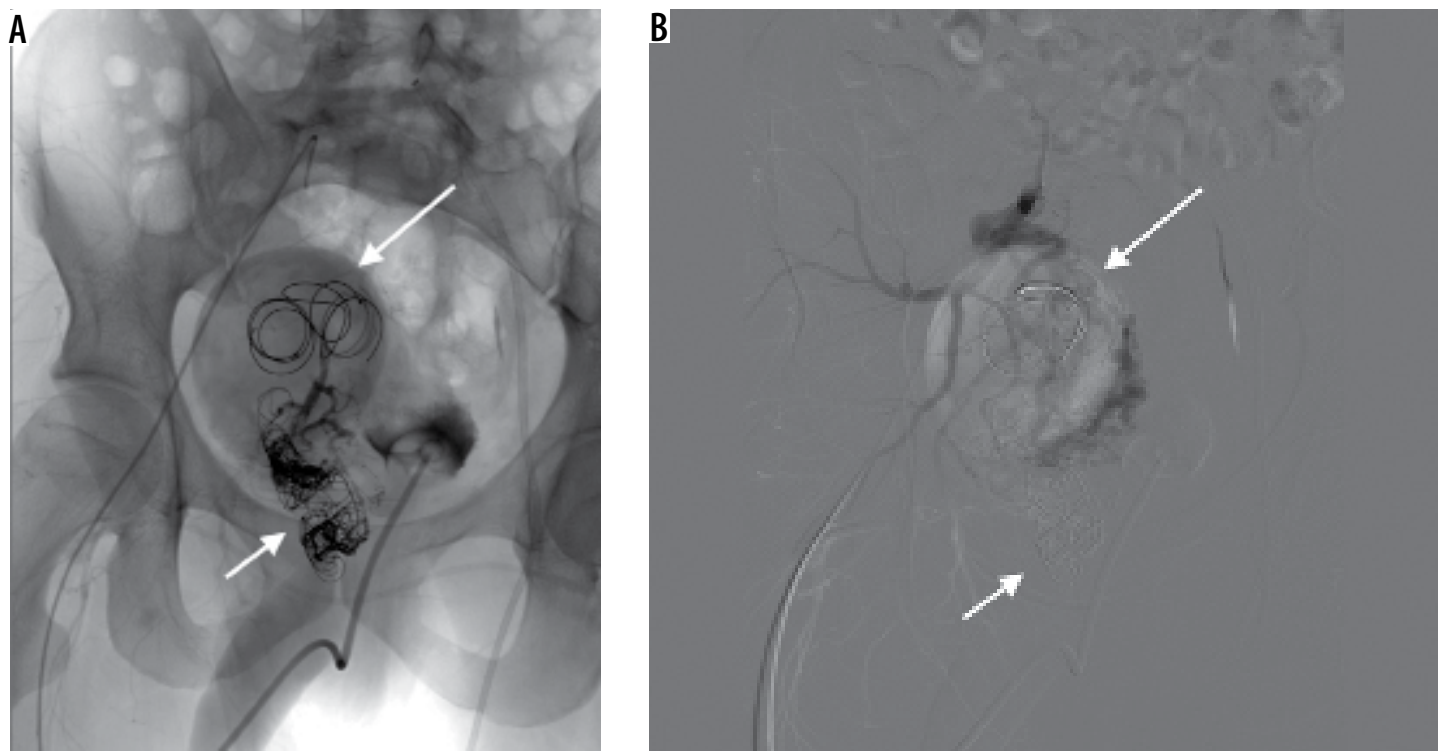

Figure 3. Non-subtracted (A) and subtracted (B) digital subtraction angiography. Detachable coils in the outflow veins (short arrow) essentially decreasing the outflow from the arteriovenous malformations. Venous aneurysm still patent (long arrow) 

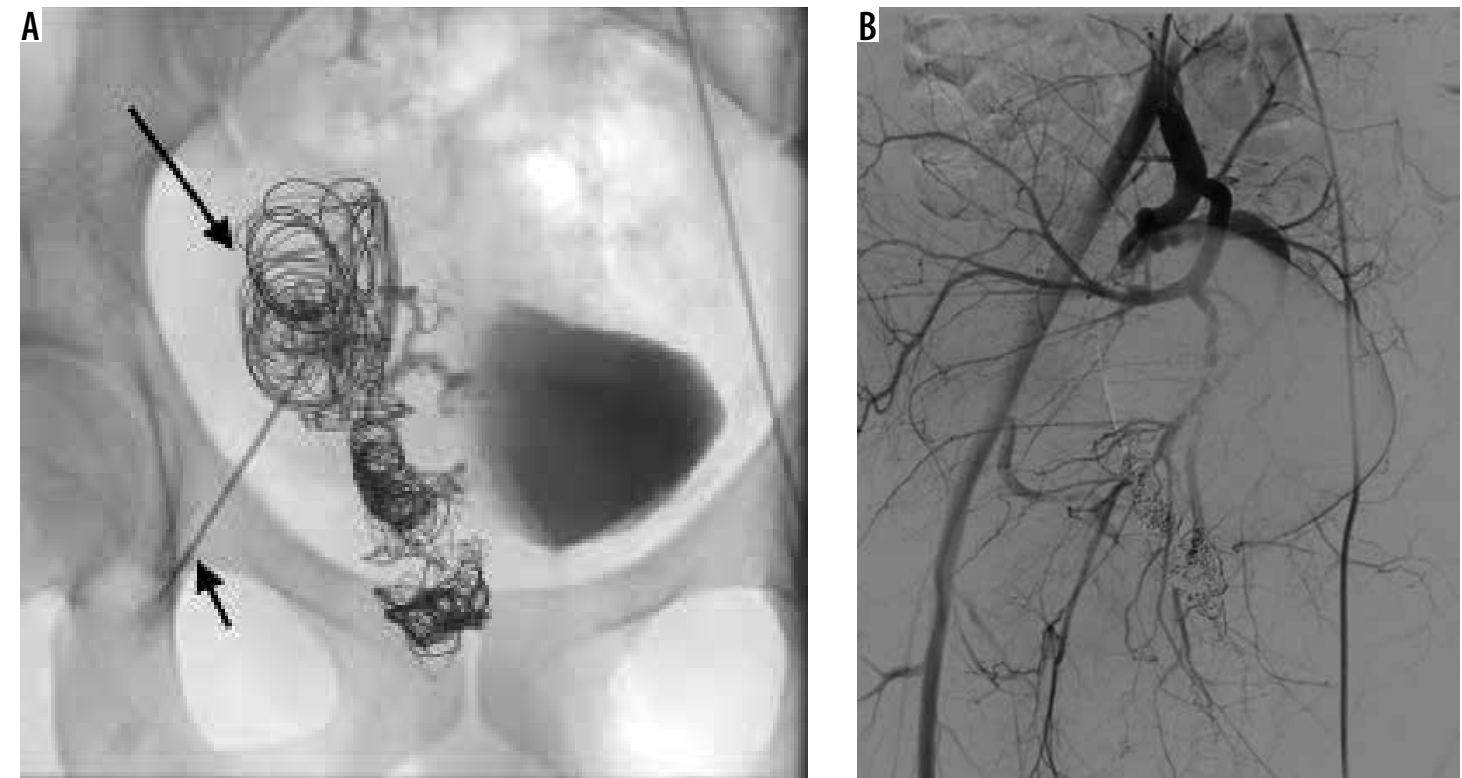

Figure 4. Direct percutaneous puncture of the venous aneurysm (short arrow) - embolisation with pushable coils and thrombin (long arrow) (A). Control digital subtraction angiography showing complete thrombosis of the venous outflow with no persisting perfusion through the arteriovenous malformations (B)

of thrombin were injected before needle removal, to improve thrombosis. A control DSA depicted minor thrombosis of the venous aneurysm and a moderate decrease in AVM perfusion, due to slower outflow through the coiled draining veins (Figure 3B).

A follow-up US performed two weeks after the procedure showed no flow in the venous aneurysm and the patient reported no pain for approximately three months until the recurrence of symptoms, which were evaluated at 7 points on the VAS. The US performed at this time revealed partial recanalisation and enlargement of the venous aneurysm.

Control DSA showed recanalisation of the AVM despite all previously implanted coils. Utilising direct puncture of the venous aneurysm (Figure 4A), six Nester coils were advanced and control DSA depicted stagnant flow in a notably diminished active zone of the aneurysm. One of the anterior trunk branches of the IAA, shown to supply the vast majority of shunts, was embolised with coils and Squid-18 Peri embolic agent. The disturbed outflow and decreased inflow finally resulted in total AVM occlusion (Figure 4B).

The patient was mildly symptomatic 3-4 days after the procedure with minor complaints of rectal prickling. Monthly US follow-ups and a final follow-up DSA after seven months showed no recanalisation of the AVM (Figure 5). To date, the patient remains asymptomatic. Doppler US follow-up at 12 and 18 months did not depict any suspicious flow spectrum in the pelvic area.

\section{Discussion}

Pelvic AVMs are composed of arteries and veins connected through a central nidus that bypasses the high-resistance

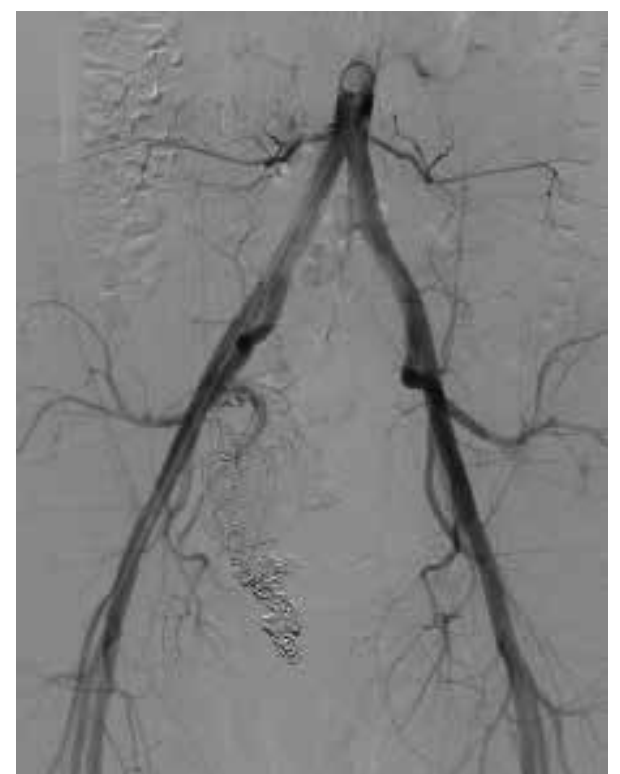

Figure 5. Follow-up digital subtraction angiography. No visible arteriovenous shunts within totally embolised arteriovenous malformation

capillary bed [1]. They display a wide spectrum of manifestations, from transient discomfort to life-threatening haemorrhagic complications [3]. Complications of untreated lesions or unsuccessful therapy include severe haemorrhagic hypovolaemic shock, cardiac failure, and cauda equina and root compression leading to diplegia and neurogenic bladder $[4,5]$. The Schobinger classification system describes their clinical course, from asymptomatic arteriovenous shunting (stage 1), through growth and functional symptoms, and finally to high-output congestive heart failure and left ventricular hypertrophy (stage 4) [6].

The treatment strategy of a pelvic AVM should be modified in consideration of the location, symptom char- 
acteristics, and the recurrence probability [1]. Although endovascular embolisation is associated with an increased rate of recurrence, it gives the possibility of additional intervention and precludes surgical resection, which is associated with an increased risk of intraoperative haemorrhage, incomplete AVM nidus removal, and a greater overall mortality rate $[2,7,8]$.

The goal of AVM embolisation is the occlusion of the nidus where there is a direct arteriovenous connection. Various patterns of peripheral AVMs are characterised by the Yakes classification, in which a certain angioarchitecture is related to the suggested embolisation approach.

The presenting pelvic AVM was classified as Yakes type IIIb, consisting of multiple feeding arteries and arterioles with a nidus in the aneurysmal vein wall with further multiple outflow veins [9]. A transvenous or percutaneous approach to the nidus area is highly indicated.
Total outflow occlusion, preferably with controlled retrograde nidus embolisation with a liquid embolic, is the most effective strategy $[9,10]$. We believe that in our case, combined arterial embolisation of the largest feeder subsequently to venous embolisation slowed down the flow within the nidus and venous aneurysm and led to total AVM thrombosis.

A thorough understanding of the vascular anatomy of the AVM is essential in choosing an effective embolisation strategy to achieve the most thorough occlusion of the pelvic AVM and to minimise the risk of possible complications.

\section{Conflict of interest}

The authors declare no conflict of interest.

\section{References}

1. Lam K, Pillai A, Reddick M. Peripheral arteriovenous malformations: classification and endovascular treatment. Appl Radiol 2017; 46: 15-21.

2. Cho SK, Do YS, Shin SW, et al. Arteriovenous malformations of the body and extremities: analysis of therapeutic outcomes and approaches according to a modified angiographic classification. J Endovasc Ther 2006; 13: 527-538.

3. Suzuki K, Tanaka N, Ebine T, et al. Pelvic congenital arteriovenous malformation diagnosed by transrectal ultrasonography: a case report. Can Urol Assoc J 2012; 6: E61-63.

4. Bekci T, Yucel S, Turgut E, et al. Giant congenital pelvic AVM causing cardiac failure, diplegia, and neurogenic bladder. Pol J Radiol 2015; 80: 388-390.

5. Donovan S, Patel N. An unusual cause of heart failure. J Cardiovasc Med (Hagerstown) 2012; 13: 399-400.

6. Kohout MP, Hansen M, Pribaz JJ, et al. Arteriovenous malformations of the head and neck: natural history and management. Plast Reconstr Surg 1998; 102: 643-654.
7. Christenson BM, Gipson MG, Smith MT. Pelvic vascular malformations. Semin Intervent Radiol 2013; 30: 364-371.

8. Mallios A, Laurian C, Houbballah R, et al. Curative treatment of pelvic arteriovenous malformation - an alternative strategy: transvenous intra-operative embolisation. Eur J Vasc Endovasc Surg 2011; 41: 548-553

9. Yakes WF, Yakes AM. Classification of arteriovenous malformation and therapeutic implication. In: Mattassi R, Loose DA, Vaghi M. Hemangiomas and Vascular Malformations: an Atlas of Diagnosis and Treatment. 2nd ed. Springer 2015; p. 263-276.

10. Wohlgemuth WA, Müller-Wille R, Teusch VI, et al. The retrograde transvenous push-through method: a novel treatment of peripheral arteriovenous malformations with dominant venous outflow. Cardiovasc Intervent Radiol 2015; 38: 623-631. 